\title{
Correction
}

\section{Correction: Silson et al., "Differential Sampling of Visual Space in Ventral and Dorsal Early Visual Cortex"}

In the article "Differential Sampling of Visual Space in Ventral and Dorsal Early Visual Cortex" by Edward H. Silson, Richard C. Reynolds, Dwight J. Kravitz, and Chris I. Baker, which appeared on pages 2294-2303 of the February 28, 2018 issue, an error in the underlying computational model was identified. This error impacted some of the estimated parameters and the fit of the model to the data. The authors apologize for the error. Rerunning the analyses with the corrected code did not affect any of the conclusions of the paper, although all of the absolute values and statistics reported have changed. All figures and numerical and statistical values reported have been corrected in the on-line PDF version. Further, the publicly available code has been corrected in the most up-to-date version of the Analysis of Functional Neuroimages software package (AFNI). The modified Results, figure legends, and figures are published below. Changed text is in bold.

\section{Results}

We used an elliptical pRF [population receptive field] model to test the prediction that ventral and dorsal divisions of EVC [early visual cortex] differentially sample visual space (Fig. 1, group average pRF parameters on the cortical surface). Before systematically comparing ventral and dorsal divisions of EVC, we established the reliability of the elliptical pRF estimates.

\section{Reliability of elliptical pRF estimates}

Initially, we compared the explained variance between the elliptical and circular pRF models using a cross-validated approach (see Reliability analyses above). On average, we observed a significant advantage for the elliptical model $\left(\boldsymbol{t}_{(\mathbf{1 1})}=\mathbf{2 . 1 0}, \boldsymbol{p}=\mathbf{0 . 0 3}\right)$, with an elliptical advantage in all but one participant, reflecting that our elliptical pRF model captures significantly more of the variance in the time courses than the circular model.

Next, we examined the reliability of our elliptical pRF parameters. For each parameter, we compared both the correlation (Pearson's) as well as the absolute differences between independent estimates (see Reliability analyses above). For each parameter, there were no significant differences in either Pearson's correlation between hemispheres [paired $\boldsymbol{t}$ test (two tailed): $\boldsymbol{x}\left(\boldsymbol{t}_{(\mathbf{1 1})}=\mathbf{0 . 2 2}, \boldsymbol{p}=\mathbf{0 . 8 2}\right)$; $y\left(t_{(11)}=0.05, p=0.95\right)$; aspect ratio $\left(t_{(11)}=0.05, p=0.95\right)$; angle $\left.\left(t_{(11)}=0.13, p=0.89\right)\right]$ or the absolute differences between hemispheres [paired $t$ test (two tailed): $x\left(t_{(11)}=0.10, p=0.91\right) ; y\left(t_{(11)}=0.23, p=0.81\right)$; aspect ratio $\left(t_{(11)}=0.06, p=0.95\right)$; angle $\left(t_{(11)}=0.73, p=0.47\right)$ ], so for further analyses we collapsed across hemispheres.

We observed significant positive correlations for all parameters $\left[\boldsymbol{t}\right.$ test relative to zero (two tailed): $x\left(t_{(11)}=42.07, p=1.66^{-13}\right)$; $y\left(t_{(11)}=33.3, p=2.13^{-12}\right)$; aspect ratio $\left(t_{(11)}=27.92, p=1.45^{-11}\right)$; angle $\left(t_{(11)}=15.84, p=6.40^{-9}\right)$; Fig. $\left.1 A\right]$. The lowest correlation was for pRF angle, but it is important to consider the influence of aspect ratio. A pRF with an aspect ratio near 1 will result in an unstable estimate of angle, as the principle axis could take a different orientation with little loss in explained variance. We therefore tested the prediction that our estimates of angle would become increasingly reliable with increasing aspect ratios. Accordingly, we computed the correlation between odd and even angle estimates for pRFs that fell into one of the following four aspect ratio bins: 1-2, 2-3, 3-4, and $4-5$ (Fig. $1 B$ ). With increasing the aspect ratio, the correlation of the angle estimates increased. A one-way repeated-measures ANOVA with aspect ratio as a within-subjects factor revealed a significant main effect of aspect ratio $\left(\boldsymbol{F}_{(\mathbf{3}, 33)}=\mathbf{2 . 8 3}, \boldsymbol{p}=\mathbf{0 . 0 5}\right)$, reflecting the increase in reliability as a function of increasing aspect ratio.

We also computed the distribution of the absolute differences in each parameter between odd and even runs. In each case, the absolute differences were small [mean $\pm \mathrm{SD}: x=0.78^{\circ} \pm 0.01^{\circ} ; y=0.73^{\circ} \pm 0.01^{\circ}$; aspect ratio $=0.05 \pm 0.07$; angle $=14.35^{\circ} \pm 1.35^{\circ}$; across all aspect ratio bins $(\mathrm{AR}): \mathrm{AR} 1-2=15.4^{\circ} \pm 1.51^{\circ} ; \mathrm{AR} 2-3=14.14^{\circ} \pm 1.35^{\circ} ; \mathrm{AR} 3-4=13.94^{\circ} \pm 1.23^{\circ} ; \mathrm{AR}^{\circ}-5=13.63^{\circ} \pm 1.3^{\circ}$ ], which reflects the reliability of our pRF parameter estimates across independent runs (Fig. $1 C$ ).

\section{Ventral and dorsal pRFs are orientated toward the fovea}

Our first prediction was that due to the restricted representations within ventral (upper visual field) and dorsal (lower visual field) divisions of EVC, and the presence of both a coarse-scale orientation map (Freeman et al., 2011) and radial biases (Sasaki et al., 2006), pRFs would exhibit a general orientation bias toward the fovea. In particular, if $0^{\circ}$ represents the horizontal axis with positive angles toward the upper vertical meridian and negative angles toward the lower vertical meridian, we hypothesized that ventral regions (V1v, $\mathrm{V} 2 \mathrm{v}$, and $\mathrm{V} 3 \mathrm{v}$ ), would show a positively biased distribution of pRF angles, with dorsal regions (V1d, V2d, and V3d) exhibiting the opposite bias, largely commensurate with their visual field representations. Our results reveal a striking difference in the distributions of pRF angle within ventral and dorsal divisions of V2 and V3 that was consistent with these predictions (Fig. 3). Initially, we tested for hemispheric differences in the distribution of pRF angle within each visual field map division using two-sample Kolmogorov-Smirnov $(\mathrm{KS})$ tests (two tailed). There were no significant differences between hemispheres for any visual field map division $[\mathbf{V 1 v}(\mathbf{K S}=\mathbf{0 . 0 7}$, $p=0.99) ; \mathrm{V} 1 \mathrm{~d}(\mathrm{KS}=0.07, p=0.99) ; \mathrm{V} 2 \mathrm{v}(\mathrm{KS}=0.07, p=0.99) ; \mathrm{V} 2 \mathrm{~d}(\mathrm{KS}=0.15, p=0.61) ; \mathrm{V} 3 \mathrm{v}(\mathrm{KS}=0.11, p=0.92) ; \mathrm{V} 3 \mathrm{~d}(\mathrm{KS}=$ $0.09, p=0.99)$ ]; thus, the distributions of pRF angle were averaged across hemispheres. Next, we compared directly the ventral and dorsal divisions for each visual field map separately using two-sampled KS tests. There were significant differences between the pRF 
angle distributions of ventral and dorsal V2 (KS $=\mathbf{0 . 1 5}, p=\mathbf{0 . 0 3})$ and V3 $(\mathrm{KS}=\mathbf{0 . 2 2}, \boldsymbol{p}=\mathbf{0 . 0 0 2})$, respectively, although ventral and dorsal V1 were not significantly different $(K S=\mathbf{0 . 0 6}, \boldsymbol{p}=\mathbf{0 . 9 8}$ ). In the case of V2 and V3, ventral maps exhibited a positively biased angle distribution, with dorsal maps exhibiting a negatively biased angle distribution largely commensurate with their visual field representations (Fig. 3).

\section{Ventral pRFs are more elliptical than dorsal pRFs}

Along with diverging orientations, we also predicted that ventral pRFs would be more elliptical than their dorsal counterparts, potentially reflecting the importance of the fovea often attributed to anterior regions of the ventral pathway and of a more even and precise representation of space in the dorsal pathway, both of which receive major input from ventral and dorsal EVC divisions, respectively (Kravitz et al., 2013). To test this, we initially computed the distribution of aspect ratios in each participant and visual field map division. First, we tested for hemispheric differences within each visual field map division using two-sample KS tests (two tailed). There were no significant differences between hemispheres for any visual field map $[\mathrm{V} 1 \mathrm{v}(\mathrm{KS}=\mathbf{0 . 0 6}, \boldsymbol{p}=\mathbf{1 . 0 0})$; V1d $(\mathrm{KS}=\mathbf{0 . 0 6}, \boldsymbol{p}=$ $1.00) ; \mathrm{V} 2 \mathrm{v}(\mathrm{KS}=0.06, p=1.00) ; \mathrm{V} 2 \mathrm{~d}(\mathrm{KS}=0.06, p=1.00) ; \mathrm{V} 3 \mathrm{v}(\mathrm{KS}=0.10, p=0.96) ; \mathrm{V} 3 \mathrm{~d}(\mathrm{KS}=0.06, p=1.00)]$; thus, distributions of aspect ratio were averaged across hemispheres (Fig. 4). Next, we compared directly each ventral and dorsal division separately using two-sampled KS tests. There were significant differences between the aspect ratio distributions of ventral and dorsal V2 $(\mathbf{K S}=\mathbf{0 . 2 4}$, $p=0.05)$ and $\mathrm{V} 3(\mathrm{KS}=0.25, p=0.03)$, but not $\mathrm{V} 1(\mathrm{KS}=0.10, p=0.47)$, despite a similar overall pattern. Across visual field maps, the distributions of aspect ratio in dorsal divisions were shifted toward smaller aspect ratios (more circular), with ventral divisions showing broader distributions.

Next, we computed the median aspect ratio in each participant and visual field map separately. Across participants, the mean aspect ratio was larger in ventral than dorsal divisions of EVC (Fig. 5A). A three-way repeated-measures ANOVA with within-participant factors of visual field map (V1, V2, V3), division (ventral, dorsal), and hemisphere (left, right) revealed a significant main effect of VFM $\left(F_{(2,22)}=41.86, p=3.16^{-8}\right)$, reflecting, on average, larger aspect ratios in V1. The main effect of division was also significant $\left(F_{(1,11)}=33.49, p=0.0001\right)$, reflecting, on average, larger aspect ratios in ventral over dorsal divisions within each region. The main effect of hemisphere was not significant $\left(F_{(1,11)}=3.25, p=0.058\right)$. No interactions were significant $(p>0.05$, in all cases). Given the nonsignificant main effect of hemisphere, we then compared directly the mean aspect ratios within ventral and dorsal divisions of EVC averaged across hemispheres using paired $t$ tests (one-tailed). Consistent with our predictions, ventral aspect ratios were significantly larger than dorsal aspect ratios in each VFM [V1v vs V1d $\left(\boldsymbol{t}_{(11)}=4.47, p=0.0001\right) ; \mathrm{V} 2 \mathrm{v}$ vs V2d $\left(\boldsymbol{t}_{(11)}=4.05, p=0.0002\right)$; V3v vs V3d $\left(t_{(11)}=5.62, p=0.00007\right.$; Fig. $\left.5 \mathrm{~A}\right]$, with a larger difference in the further anterior regions.

\section{Ventral pRFs cover larger area of visual space than dorsal pRFs}

Although we observe an increase in aspect ratio for ventral pRFs, it is important to consider the area of visual space covered by these elliptical pRFs. An increase in aspect ratio alone could reflect either elongation of the major axis or shrinking of the minor axis and thus an overall reduction in area or elongation of the major axis and an enlarging of overall area. To distinguish between these two possibilities, we calculated the median area in each participant and visual field map, respectively. On average, pRF area was larger in ventral compared with dorsal divisions of EVC (Fig. $5 B$ ). A three-way repeated-measures ANOVA (VFM, division, and hemisphere) revealed significant main effects of both $\operatorname{VFM}\left(F_{(2,22)}=37.89, p=7.47^{-8}\right)$ and division $\left(F_{(1,11)}=11.30, p=0.006\right)$. However, these main effects are qualified by a significant VFM by division interaction $\left(F_{(2,22)}=17.41, p=0.00003\right)$, which highlights that the magnitude of the difference between ventral and dorsal divisions varies across regions. No other interactions were significant $(p>0.05$, in all cases). Given the nonsignificant main effect of hemisphere $\left(\boldsymbol{F}_{(\mathbf{1}, 11)}=\mathbf{2 . 2 2}, \boldsymbol{p}=\mathbf{0 . 1 6}\right)$, pRF area values were averaged across hemispheres. Again, three separate two-way ANOVAs were computed to better interpret the above interaction. These analyses revealed a larger ventral/dorsal difference in V3 compared with both V1 and V2 [V1-V2: VFM by division $\left(\boldsymbol{F}_{(1,11)}=\mathbf{2 . 3 5}, \boldsymbol{p}=\mathbf{0 . 1 5}\right)$; V1-V3: VFM by division $\left(F_{(1,11)}=13.40 p=0.004\right)$; V2-V3: VFM by division $\left.\left(F_{(1,11)}=\mathbf{3 0 . 4 8}, p=0.0001\right)\right]$. Next, we compared directly the $\mathrm{pRF}$ area within ventral and dorsal divisions of each visual field map using paired $t$ tests (one tailed). Ventral pRFs covered a significantly larger area of the visual field than dorsal pRFs in V1 and V3 [V1v vs V1d $\left(\boldsymbol{t}_{(11)}=3.37, \boldsymbol{p}=\mathbf{0 . 0 0 3}\right)$; $\mathrm{V} 3 \mathrm{v}$ vs V3d $\left(\boldsymbol{t}_{(11)}=4.27, \boldsymbol{p}=\mathbf{0 . 0 0 0 5}\right)$ ], but not in $\mathrm{V} 2\left[\mathrm{~V} 2 \mathrm{v}\right.$ vs $\left.\mathrm{V} 2 \mathrm{~d}\left(\boldsymbol{t}_{(11)}=\mathbf{0 . 9 1}, \boldsymbol{p}=\mathbf{0 . 1 9}\right)\right]$, although the numerical increase trended toward significance.

\section{pRF area and aspect ratio increase with increasing eccentricity}

Until now, our analyses have focused on isolated parameters (e.g., angle, aspect ratio, or area), but it is also important to consider the relationship between parameters. It is commonly reported that the size of receptive fields (or pRFs) increases with increasing eccentricity, and that this increase is often linear (Hubel and Wiesel, 1974; Maunsell and Newsome, 1987; Dumoulin and Wandell, 2008; Winawer et al., 2010). Therefore, we investigated the relationship between eccentricity and both pRF area and aspect ratio, which we predicted to show a similar near linear relationship. For each participant and visual field map we calculated (1) the median area and (2) the median aspect ratio of pRFs in bins of $1^{\circ}$ of eccentricity. Next, we computed the average pRF values within each bin collapsing across hemispheres (Fig. 6A,B).

The largely near linear trend between eccentricity and pRF size reported previously (Dumoulin and Wandell, 2008; Winawer et al., 2010 ) is evident in the estimates of both pRF area (Fig. 6A) and aspect ratio (Fig. 6B). Indeed, the general tendency for larger pRFs within successive visual field maps at equal eccentricities is also present for area, with V3 area estimates being larger than those of V2 and V1, respectively (Fig. 6A). Notwithstanding differences in absolute aspect ratio (Fig. 6B), all regions exhibit a similar near linear relationship whereby aspect ratio increases as a function of eccentricity. 


\section{Differential sampling of space between ventral and dorsal EVC not due to differences in explained variance}

While we observe systematic differences between pRFs in ventral and dorsal divisions of EVC, it is important to rule out the possibility that these differences are due to systematic differences in the explained variance of our model between these ventral and dorsal divisions. To test this, we calculated the median explained variance in each participant and visual field map, respectively. A three-way repeated-measures ANOVA (VFM, division, and hemisphere) revealed only a significant main effect of VFM $\left(\boldsymbol{F}_{(\mathbf{2}, \mathbf{2 2})}=\mathbf{8 . 2 4 1}, \boldsymbol{p}=\right.$ 0.002), reflecting, on average, the larger explained variance in $\mathrm{V} 3$ (mean $\pm \mathrm{SD}: \mathrm{V} 1=0.56 \pm 0.08 ; \mathrm{V} 2=0.59 \pm 0.10 ; \mathrm{V} 3=0.61 \pm 0.10)$. All other main effects and interactions were not significant $(p>0.05$, in all cases). Thus, the systematic differences reported here between ventral and dorsal EVC are not due to poorer model fits in one division over the other.

\section{Summary comparison of circular and elliptical pRF models}

Finally, to summarize and exemplify the additional information provided by the elliptical pRF model, we calculated the average pRF parameters $(x, y$, aspect ratio, and angle) in V2d and V2v (in the left hemisphere) across participants using both circular and elliptical pRF models. A visualization of the resulting average pRFs (Fig. 7) demonstrates the additional information of the elliptical model. In both models, the centers $(x, y)$ of each pRF are largely equivalent, indicating that the addition of parameters for aspect ratio and angle made little difference to the center of the pRF. However, allowing the shape of the pRF to vary not only captures the greater elongation of ventral pRFs, but also demonstrates that this elongation is accompanied by an increase in the area of covered visual field. The orientation of both pRFs toward the fovea is also evident and cannot be captured by a strictly circular model.

Group average $n=12$
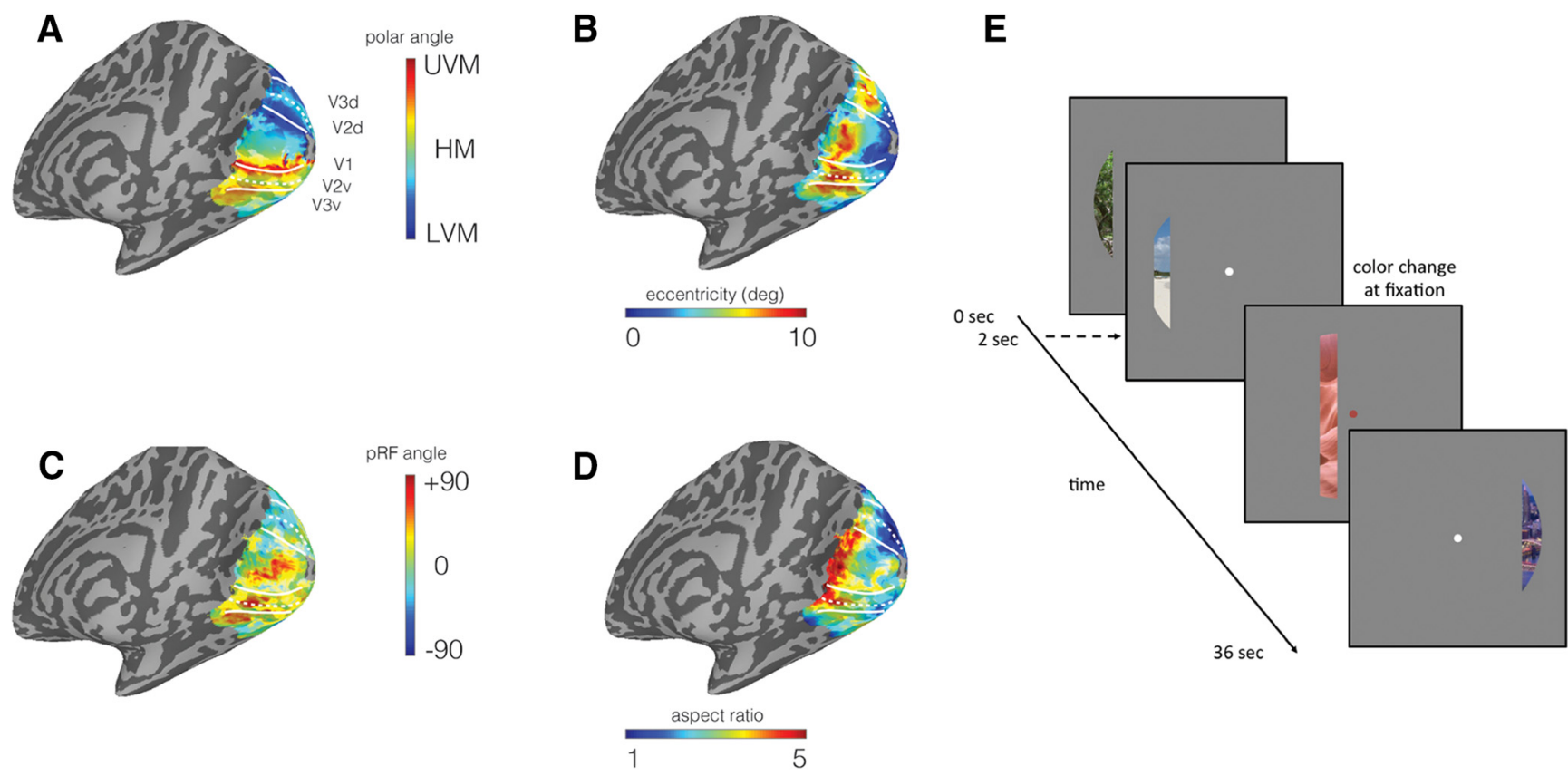

Figure 1. 
A

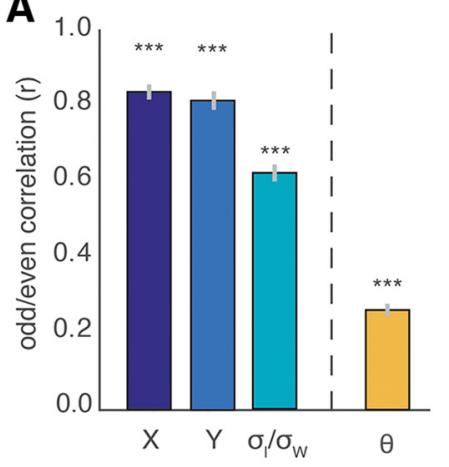

C
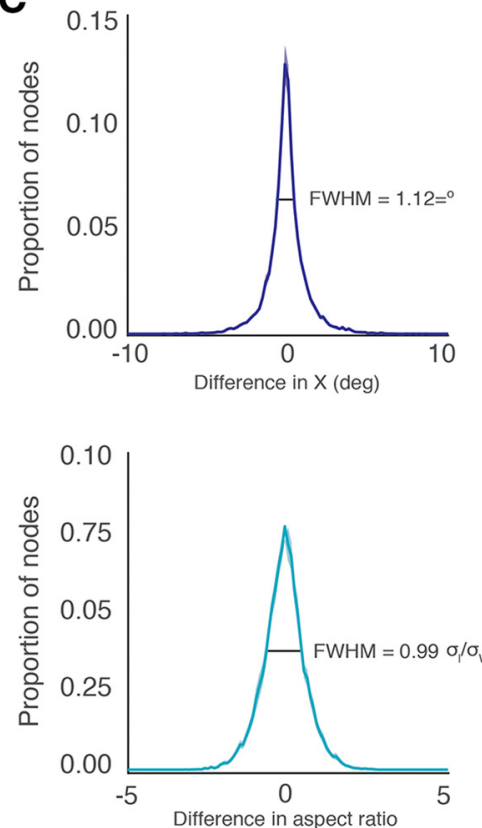

B
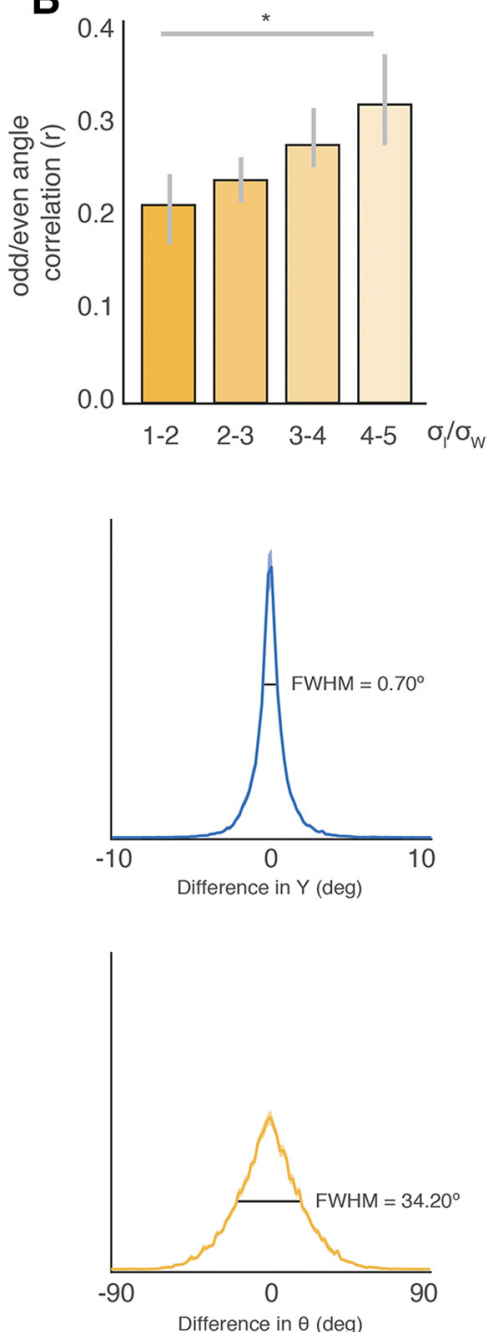

Figure 2. Reliability of the elliptical and oriented pRF model. A, Correlation values derived between independent sets of data for each of the parameters of interest. We observe significant positive correlations for all parameters of interest. The dashed vertical line separates our angle estimate from the other parameters, because the reliability of this parameter was calculated using a circular correlation method ${ }^{* * *} p<0.001$, relative to zero). $\boldsymbol{B}$, Reliability of theta estimates as a function of aspect ratio. We tested the prediction that our theta estimates would become increasingly reliable and aspect ratio increased, as a pRF with an aspect ratio of 1 will result in an unstable theta estimate. As predicted, a one-way repeated-measures ANOVA confirmed that the reliability of our theta estimates increased with increasing aspect ratio ( ${ }^{*} \boldsymbol{p}<\mathbf{0 . 0 5}$ for the main effect of aspect ratio). C, Distributions of the absolute differences between odd/even runs for each parameter. For each distribution, the FWHM is shown by the black line with the value given to the right. 
A

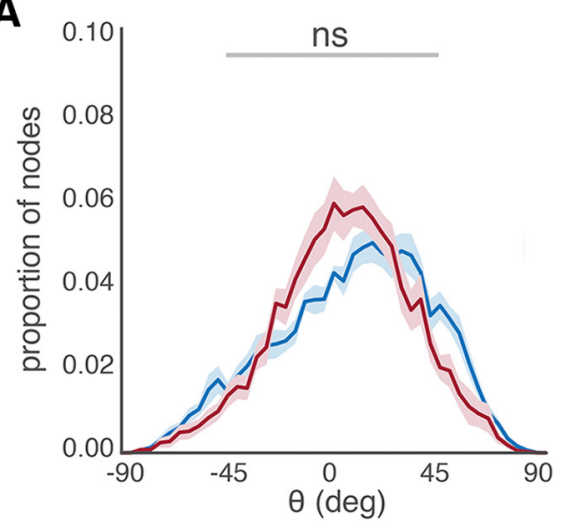

B

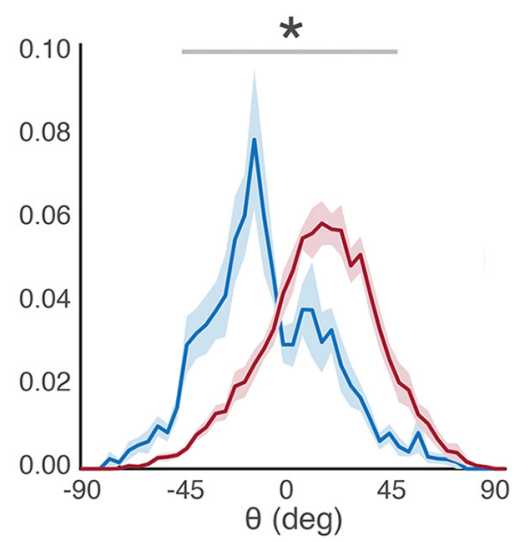

C

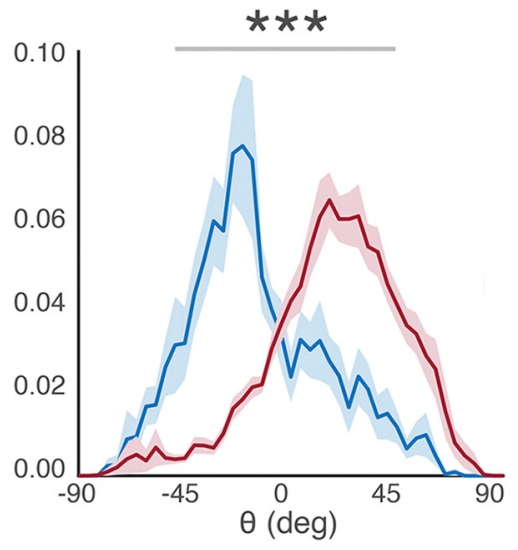

90

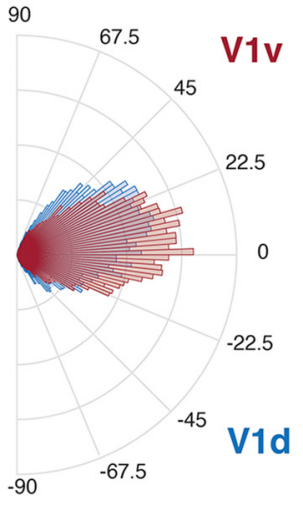

90

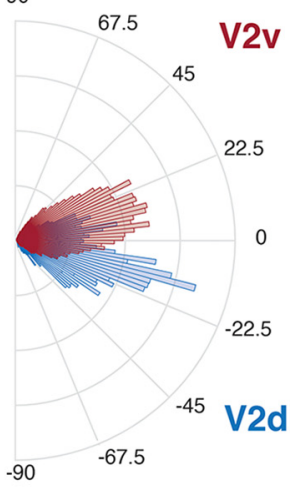

90

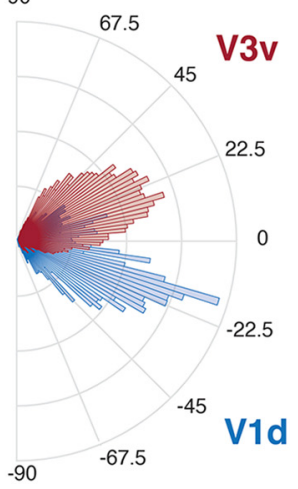

Figure 3. pRF orientation biases in dorsal and ventral divisions of EVC. $A$, Distributions of pRF orientation in V1d (blue line) and V1v (red line) collapsed across participants and hemispheres. Both distributions appear to be centered largely around zero (horizontal), with little difference in overall bias. The angle histograms of both distributions are plotted to the right. $\boldsymbol{B}$, Distributions of pRF orientation in V2d (blue line) and V2v (red line) collapsed across participants and hemispheres. V2d exhibits a distribution shifted toward negative orientations, whereas V2v exhibits the opposite bias. These distributions were significantly different from one another (two-sample KS test). The angle histograms of both distributions are plotted to the right and better depict the differential pRF angles in both regions. C, Distributions of pRF orientation in V3d (blue line) and V3V (red line) collapsed across participants and hemispheres. V3d exhibits a distribution shifted toward negative orientations, whereas V3v exhibits the opposite bias. These distributions were significantly different from one another (two-sample KS test). The angle histograms of both distributions are plotted to the right and better depicts the differential pRF angles in both regions. ${ }^{*} p<0.05,{ }^{* * *} p<0.001$. 

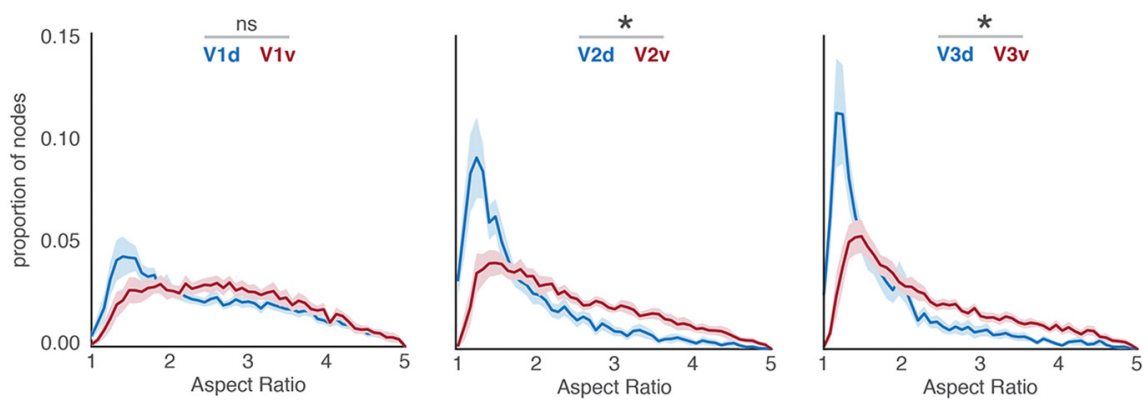

Figure 4. pRF aspect ratio distributions in ventral and dorsal divisions of EVC. Plots depict the average distributions of aspect ratio in each visual field map and division, respectively $($ blue line $=$ dorsal; red line = ventral). In each case, dorsal distributions are shifted toward smaller aspect ratios. Distributions within each visual field map were compared using two-sample KS tests. In each visual field map of $\mathrm{V} 2$ and $\mathrm{V} 3$, ventral and dorsal distributions were significantly different from one another $\left({ }^{*} p<0.05\right)$.

A

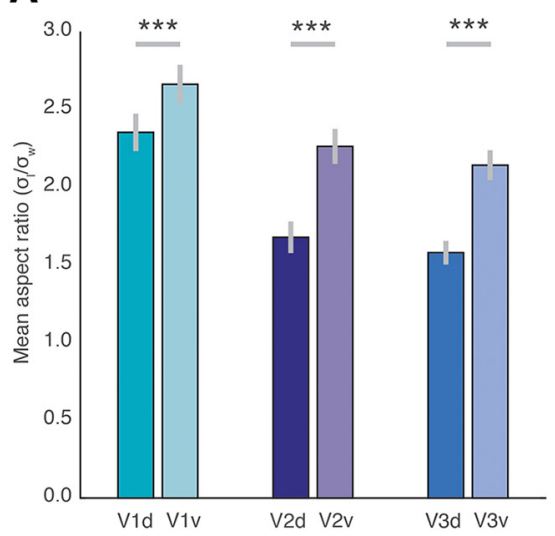

B

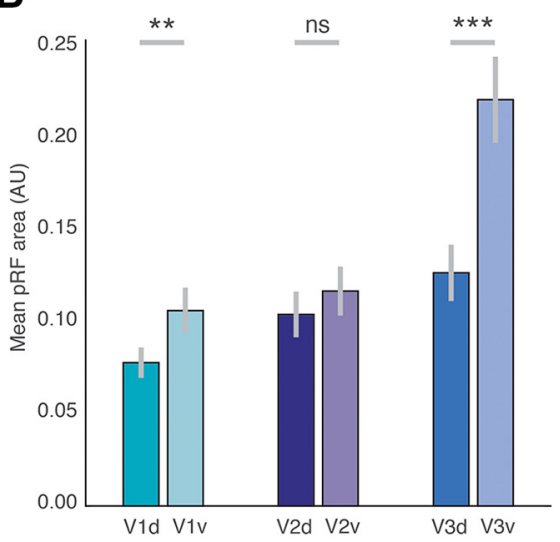

Figure 5. Dorsal versus ventral comparisons. $\boldsymbol{A}$, Bars represent the mean of the median aspect ratio in each visual field map division collapsed across participants and hemispheres. pRF aspect ratio was significantly larger within the ventral division of each visual field map (paired $t$ test between dorsal and ventral divisions). $\boldsymbol{B}$, Bars depict the mean of the median pRF area in each visual field map division collapsed across participants and hemispheres. pRF area was nominally larger in ventral over dorsal V2, and significantly larger in ventral over dorsal V1 and V3 (paired $t$ test between dorsal and ventral divisions). ${ }^{* *} p<0.01,{ }^{* * *} p<0.001$.

A

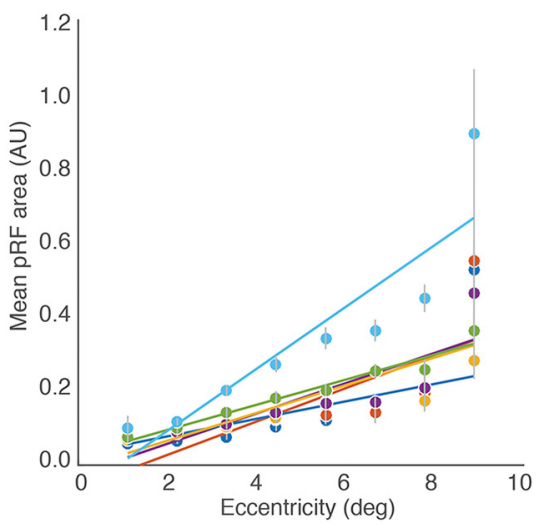

B

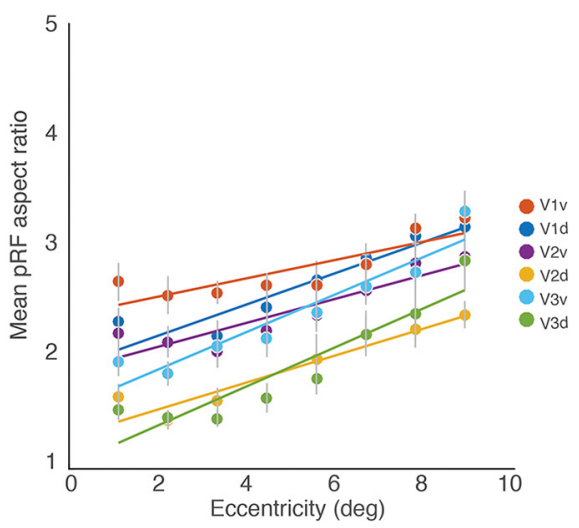

Figure 6. 


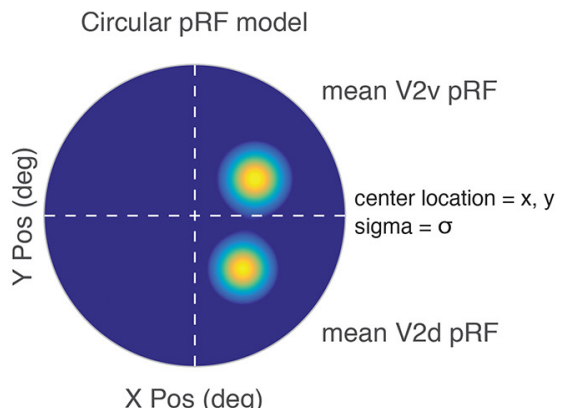

Elliptical pRF model

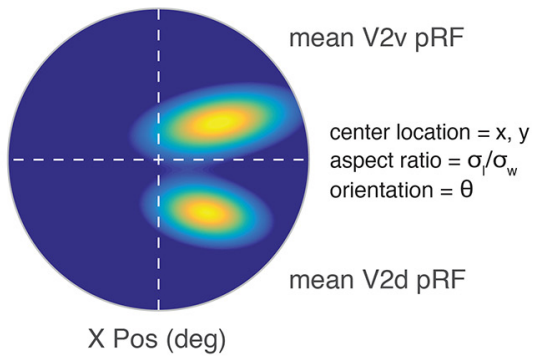

Figure 7. 